\title{
ILP BASED EVALUATION OF SEPARATE WAVELENGTH POOL (SWAP) STRATEGY
}

\author{
Zsolt Lakatos Ph.D. Student \\ Budapest University of Technology and Economics, Department of Telecommunications \\ Magyar Tudósok krt 2, Budapest, Hungary H-1117,e-mail:lakatos@hit.bme.hu
}

\begin{abstract}
The paper proposes a general approach to support the analysis and evaluation of dynamic routing and wavelength assignment strategies applicable in advanced optical networks. Some ILP based models are introduced to apply the proposed analysis support for the evaluation of Separate Wavelength Pool strategy. Results from a small network example are described and analyzed to validate the proposed ILP models in one hand and to highlight the advantages of the shared capacity related resilience and the preemption techniques applicable in advanced optical networks on the other hand.
\end{abstract}

\section{INTRODUCTION}

Recent developments in optical technology enable the implementation of complex network and management functionalities. The simple dynamic routing and wavelength assignment (RWA) strategies can be replaced by more complex and effective ones based on the advanced signaling.

Due to the various client requirements the optical network layer should support differentiated transport services. In case of dynamic optical networks this differentiation can be made according to the blocking probabilities and resilience options of the arriving optical channel requests[1].

Taking into account the typical IP client requirements two basic service classes can be distinguished in the optical layer: the premium leased optical channel service for high quality traffic (e.g. IP VPNs, QoS oriented applications, etc.); the low priority leased optical channel service for IP links carrying best-effort traffic.

The premium class is with low blocking probability and guaranteed protection in case of network element failures, however, there is no bounds for the blocking of low priority class. Even the optical channels carrying the traffic of the low priority class are pre-emptable in case of network element failures in order to support the guaranteed protection of the premium class. In case of the application of different QoP classes - mainly if a pre-emptable class is present - the availability 
analysis of different service classes provide further important information on the provisioning strategy[2].

In case of dynamic optical channel provisioning the permanent optical channel requests are assumed to arrive spread in time and space, and an appropriate strategy is applied to meet them under the efficient utilization of available network resources. Having these two service classes the main goal of a provisioning strategy is to restrict the uncontrolled competition between the premium and low priority traffic, since the implementation of the distinction in blocking probabilities requires efficient control during the allocation of the optical channels for requests from different classes.

\section{EVALUATION OF RESILIENT OPTICAL CHANNEL PROVISIONING STRATEGIES}

To evaluate resilient optical channel provisioning strategies three aspects should be taken into account: penalty on dynamic behavior, complexity of required network consolidations, availability of low priority services.

Generally, solving a provisioning problem a random order of optical channel requests is to serve. It results in a sub-optimal decision for resource allocation, since even in case of pre-planned fixed routing the wavelength assignment is performed channel by channel subsequently according to a simple strategy (e.g. first fit). Having known the same set of optical channels in advance and routed them via the same routes an optimal solution for the wavelength assignment under fixed routes can be achieved, and the difference in the required resources can be interpreted as a penalty for the dynamic behavior. With other words this difference describes how far a random sequence based solution (or the average of numerous solutions) from the theoretically achievable global optimum.

To illustrate the above described penalty on dynamic behavior results from a small ( 9 nodes, 16 edges, nodal degree 3.5) network example is presented in Figure 1[3]. The left bar gives the average resource needs in case of sequential allocation, the right one represents the global optimum. The demand patterns and the routing of the optical channels are the same in both cases, the only difference is in the wavelength allocation. In the sequential case it is a simple first fit strategy [4] (sequential allocation of the idle - not yet allocated - optical channels via the fixed route), in the reference case it is an optimal - requiring minimum number of wavelengths - solution based on the ILP implementation of the graph-coloring model. The lower parts of the bars represent the amount of optical channels in use; the upper part is the amount of extra wavelengths to be installed in case of no wavelength conversion. The figures represent the total number of wavelength on links. It can be depicted on the figure that the sequential first fit allocation results in additional $30 \%$ wavelength need. It is a theoretical lower bound, since there may not be a proper sequence for a given dynamic allocation strategy to achieve this 
lower bound. However, the distance between the theoretical bound and the resource need for a given strategy is a good measure for the evaluation.

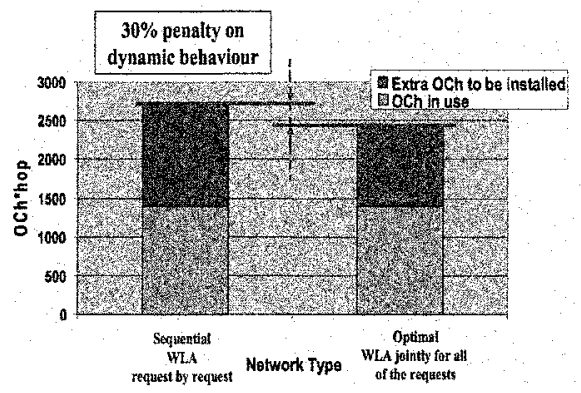

Figure 1 Penalty of dynamic behavior

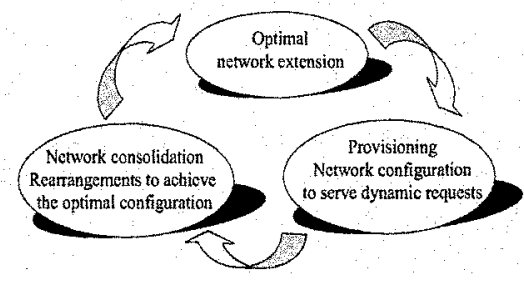

Figure 2 Extension - Provisioning Consolidation scheme of the network lifecycle

If we change our focus from the simple provisioning problem to the network development, an interesting problem can be identified. Since the traffic to be served supposed to be incremental (permanent channel requests with practically infinite holding time) the saturation of network resources are foreseen. In this case based on the experienced structure and amount of channel requests the network resources should be extended to prevent the significant increase of blocking. On the other hand, as we have seen from the previous illustration the network configuration is sub/optimal due to the channel by channel sequential solution of RWA problem. Thus, it is a quite obvious idea to consolidate the network in order to improve the resource utilization before the decisions on the capacity extension.

Figure 2 illustrates a simple Extension - Provisioning - Consolidation scheme of the network lifecycle. The network consolidation means a network reconfiguration (rearrangement) which from technological aspects can be based on the same automatic configuration capabilities as the provisioning.

The general green-field network optimization problem can be formalized as follows: the network topology is given as a $G(V, E)$ with sets of nodes $V$ and edges $E$ without effective capacity constraints. Edge $n, m$ is assigned to cost function $\Phi_{m, n}\left(C_{m, n}\right)$, where $C_{m, n}$ is the required capacity on the given edge. The network is have to meet demands $D=\left\{d_{i j}\right\}$ under minimum cost $\phi(\underline{C})=\sum \phi_{m, n}\left(C_{m, n}\right)$. The edge capacities can be derived from the demands according to the applicable routing and the given topology.

The network consolidation problem can be formalized differently: in this case a complete network configuration is given initially, with its topology $G(V, E)$, demands $\mathrm{D}=\left\{\mathrm{d}_{\mathrm{ij}}\right\}$, and routing of demands $\mathrm{P} 0=\left\{\mathrm{p} 0_{\mathrm{i}, \mathrm{j}}\right\}$, link capacities $\mathrm{C}(\mathrm{m}, \mathrm{n})$ for each link $m, n$. The aim of the consolidation to optimize the utilization of the existing resources (link capacities) with limited network rearrangements. Therefore, the network is have to meet demands $\mathrm{D}=\left\{\mathrm{d}_{\mathrm{i}, \mathrm{j}}\right\}$ under maximum saving $\phi\left(\underline{C}^{0}-\underline{C}\right)=\sum \phi_{m, n}\left(C_{m, n}^{0}-C_{m, n}^{*}\right)$. To improve the basic model some 
penalties on the network rearrangements decreasing the savings can be taken into account. The penalty may depend on the extend of changes of the applied routes $\Pi\left(\mathrm{P}^{0}, \mathrm{P}^{*}\right)$, thus the complete target function to be maximized is $\phi\left(\underline{C}^{0}-\underline{C}\right)$ $\Pi\left(\mathrm{P}^{0}, \mathrm{P}^{*}\right)$. Depending on the applied network model link capacity $C_{m, n}$ may represent wavelengths or wavelength multiplex modules in both cases.

\section{THE SWAP STRATEGY AND SOME ILP MODEL FOR ITS EVALUATION}

When there are different traffic classes to be supported in optical channel provisioning strategies, it is a general problem how to protect the premium class traffic from the uncontrolled competition with the low priority traffic. Separate Wavelength Pool strategy gives a simple and effective solution for this problem [5]. The available wavelength pool is separated in SWAP, the first pool serves to allocate working connections for premium traffic only, and the second pool is for SRLG-based shared spare capacity oriented restoration routes for premium class traffic and for working routes of low priority pre-emptable traffic. Assuming that the premium traffic can be forecasted with acceptable level of confidence, and based on this forecast the available wavelength pool can be shared. This resource sharing guarantees the high quality service (guaranteed protection, low blocking) of premium traffic independently from the amount and pattern of the low priority traffic (which assumed to be hardly forecastable).

ILP based modelling of the SWAP strategy helps to study the main features of the solution independently from the impact of random optical channel request arrival sequences. Having an assumed demand pattern the amount and configuration of required resources can be calculated to serve the given pattern or having both the network and the demands the feasibility of the specified problem can be checked.

The general ILP approach to study SWAP strategy is based on the minimum cost flow model. To different network models can be supported applying different target functions. When the target is to minimize the total amount of used optical channels it is the single fiber model (no modularity of wavelength multiplex systems is concerned). Specifying the total amount of wavelength multiplex systems to be minimized the modular network model is implemented. (In the latest case the capacity of the wavelength multiplex system in wavelengths should be specified.) The applied model is a real flow based one, where Kirchoff's laws are applied to control the flows in the source, sink and intermediate nodes. The first law assures that the given flow leaves the source, the second that it arrives to the sink, and the third is the flow conservation in the intermediate nodes.

The resilience for premium class connection is provided via a route disjoint from the working one. This approach simplifies the provisioning processes since 
the same restoration route can be applied in case of any single link failure. This constraint is implemented by (1)

$$
\sum_{\forall h}{ }^{h} W_{m, n}+\sum_{\forall h^{*}}{ }^{h^{*}} P_{m, n} \leq 1 \quad W, P \in\{0,1\} \quad \text { (1) where } W_{m, n} \text { and } P_{m, n}
$$

indicate the usage of $h$ working and $h^{*}$ protection wavelengths on link $n, m$.

Applying the modular network model, the number of modules should be

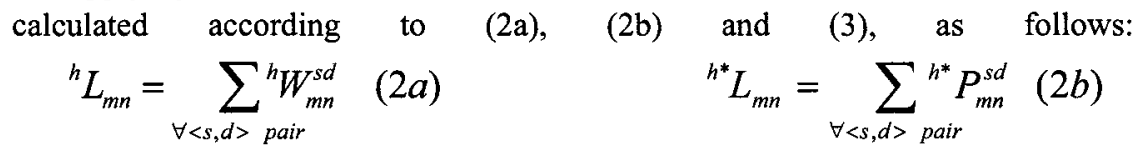

$$
M_{m n}=\max _{\forall\left(h \cup h^{*}\right)}{ }^{h} L_{m n} \quad \text { (3) where }{ }^{h} W_{m, n}^{s, d} \text { indicates that the working route of }
$$

optical channel demand between s,d routed via link $\mathrm{n}, \mathrm{m}$ on wavelength $\mathrm{h},{ }^{h^{*}} P_{m, n}^{s, d}$ is the same for the protection route on wavelength $h^{*}$. Accumulating these information ${ }^{h} L_{m, n}$ and ${ }^{h^{*}} L_{m, n}$ give the multiplicity of wavelengths $\mathrm{h}$ and $\mathrm{h} *$ for link $\mathrm{m}, \mathrm{n}$, respectively, and larger multiplicity sets the number of required modules on the link n,m.

With help of these basic formulas four variations are specified to model different network and technology scenarios.

The first scenario (referred later as scenario " $\underline{\underline{I} \boldsymbol{I}}$ ") is specified for reference purposes only. The premium traffic is routed according to the traditional $1+1$ dedicated protection. The protection routes are calculated under fixed working routes and the wavelengths are assigned to them from the protection pool. Comparing the results from this case with the results with shared capacity oriented resilience, the efficiency of resilience oriented capacity sharing can be studied (referred later as $1+1$ ). (No low priority traffic is routed in this case.)

The second scenario (referred later as scenario "shared") is dedicated to the shared capacity based resilience. Minimum disjoint paths are calculated to the fixed working ones for the premium traffic, and the spare capacities allocation is based on to the Shared Risk Link Groups (SRLG) set up according to the working routes. The calculation of spare capacities are performed according to formulas (4) and

$h^{*} F_{m n}^{i j}=\sum_{\forall<s, d>} h^{*} P_{m n}^{s d}$

$$
{ }^{h^{*}} L_{m n}=\max _{<i, j>} h^{h^{*}} F_{m n}^{i j}
$$

where, due to the capacity sharing the failure case should be identified - $i_{2}$ j denote the failed link, and ${ }^{*} F_{m, n}^{i, j}$ represents the multiplicity of wavelength $\mathrm{h}^{*}$ in use on link $\mathrm{m}, \mathrm{n}$ in case of the failure of link $\mathrm{i}, \mathrm{j}$. The maximum of ${ }^{h^{*}} F_{m, n}^{i, j}$ taking into account each failure case gives the required multiplicity of the wavelength $h^{*}$ on link m,n, and is represented by ${ }^{h^{*}} L_{m, n}$. (No low priority traffic routed in this case.) 
The third scenario (referred later as scenario "pre-empe") is the extension of the previous one to include low priority traffic, as well. The premium traffic is routed according to the disjoint path SRLG based shared capacity resilience option described above, and the low priority traffic is via minimum paths and the wavelengths are assigned to the paths from the second pool. Low priority traffic is pre-emptable; thus it may use the spare resources of the high priority traffic. Therefore, the Kirchoff's laws should be formulated for low priority traffic as well, and the calculation of required wavelengths should be modified according to (6), where the larger of the premium shared protection of low priority working requirements will set up the multiplicity of a wavelength $h^{*}$ on a given link m,n.

$$
{ }^{h^{*}} L_{m n}=\max \left\{\max _{<i, j>}{ }^{h^{*}} F_{m n}^{i j}, h^{*} L_{m n}^{\prime}\right\}
$$

The fourth scenario (referred later as scenario " $\underline{a d d}$ ') is differs from the third one in the resource allocation for low priority traffic. In this case the low priority traffic is not pre-emptable, therefore its resource needs are additional in the second pool. Formula (7) gives the related calculation of the required multiplicity of wavelengths in the second pool on a given link.

$$
{ }^{h^{*}} L_{m n}=\max _{<i, j>}{ }^{h^{*}} F_{m n}^{i j}+{ }^{h^{*}} L_{m n}^{\prime}
$$

The differences between formulas (6) and (7) express the impact of preemptable traffic, since in (6) the maximum in (7) the sum set up the required multiplicity of wavelengths.

\section{ILLUSTRATIVE EXAMPLES}

In the chapter some illustrative examples are presented to validate the proposed study approaches and the elaborated ILP models. The network example applied for the illustrations is a $3 \times 3$ mesh-torus, which is a regular topology with uniform nodal degree and routing capabilities. The applied traffic pattern is a uniform full mesh one, both for premium and low priority traffics. The illustrations are based on a modular network model, the capacity of a wavelength multiplex system is four wavelengths, equally shared between the first and the second pool. There are no wavelength conversion functions assumed in the nodes, however, flexible wavelength selection function are assumed on the ingress sides, thus the assignment of the available wavelengths to the connections are without any restrictions. The small illustrative studies are focused on the calculation of resource needs in different scenarios. The general approach that the optical channel requests are supposed to be known in advance and the minimum amount of resources required serving them can be calculated.

Figures $3 \mathrm{a}$ and $3 \mathrm{~b}$ depict the resource needs in terms of total amount of required wavelengths and wavelength multiplex modules for different scenarios optimized for the number of wavelengths and number of wavelength multiplex modules required. Comparing the corresponding results established for $1+1$ dedicated and shared protection $17 \%$ gain in number of required modules, and $40 \%$ gain in 
number of total required wavelengths could be identified due to the spare capacity sharing.

Performing the similar comparisons for scenarios with additional (add.) and preemptable (pre-empt.) handling of low priority traffic, $15 \%$ gain in number of required modules, and $17 \%$ gain in number of total required wavelengths can be identified due to the pre-emption.

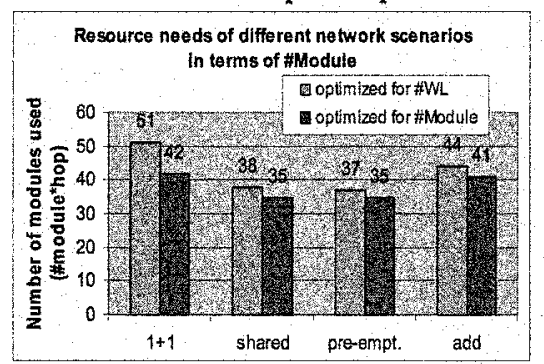

Figure 3 a Resource needs of different network scenarios in terms of \#Module

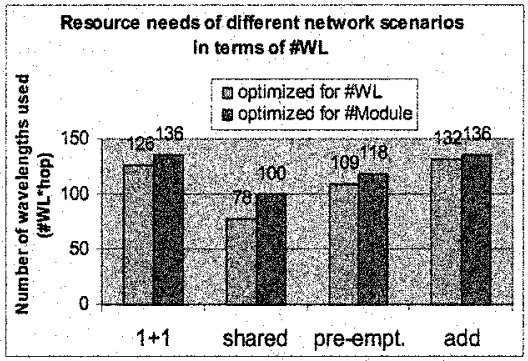

Figure $3 b$ Resource needs of different network scenarios in terms of \#WL

The tendencies are met the initial expectations in both cases, and the differences in number of wavelengths and in number of modules in case of the two optimization approaches - minimum total used wavelengths, minimum total used modules - are according to the general expectations, as well.

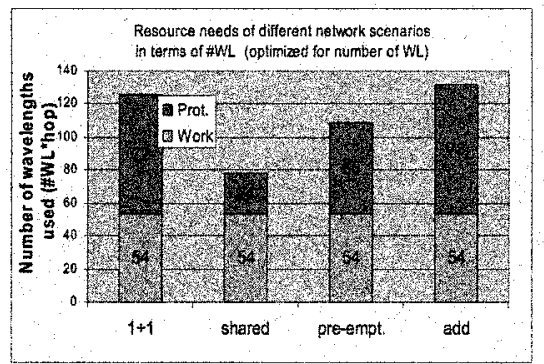

Figure 4a Resource needs of different network scenarios optimized for \#WL

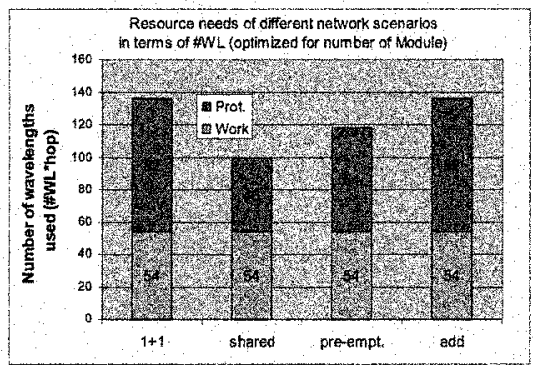

Figure $4 b$ Resource needs of different network scenarios optimized for \#Module

Figures $4 \mathrm{a}$ and $4 \mathrm{~b}$ give the breakdown of the resource needs depicted in Figure $3 \mathrm{~b}$. Based on the results of Figure $4 \mathrm{a}$ the resource savings both in terms of wavelengths and modules can be analyzed in details. Due to the same working routes of premium traffic, the wavelengths required in the first pool are the same in all cases (lower parts of the bars). The extra resource needs dedicated to the resilience of premium traffic can be depicted, as well (upper parts of the bars). Analyzing saving of the resilience related resources due to the spare capacity sharing and pre-emption $-66 \%$ and $30 \%$, respectively- the main positive features of the SWAP strategy can be identified more clearly.

Concerning the validation of the applied ILP models besides the confirmation of the expected tendencies, and additional cross-checking can be made. Based on the results on Figure 4a the working routes of the premium traffic require 54 
wavelengths in total (lower part of the bar for scenario "shared"). The shared capacity oriented restoration of these working routes requires 24 additional wavelengths (upper part of the bar for scenario "shared") taking into account single link failures and guaranteed restoration. Note that in scenario "shared" there is no low priority traffic routed in the network. Besides the protected premium traffic in scenario "add" low priority traffic of same pattern as the premium one is routed in the network, and requires 54 wavelengths in total in the second pool. Since the resource needs of low priority traffic are considered as additional, the total wavelengths required in the second pool in scenario "add" could be interpreted as follows: 54 wavelengths for working routes of premium traffic (lower part of the bar for scenario "shared"), 54 wavelengths for working routes of low priority traffic plus 24 wavelengths for shared capacity oriented restoration of premium traffic, which makes 78 wavelengths in total (upper part of the bar for scenario "shared").

\section{SUMMARY AND CONCLUSIONS}

A general approach is described to study and evaluate dynamic routing and wavelength assignment strategies applicable in advanced optical networks. Based on the proposed approach some ILP based models are proposed to provide reference results for the evaluation of Separate Wavelength Pool strategy. The illustrative results validate the proposed models, and despite of the small size of the studied example the results highlight the advantages of the shared capacity related resilience and the pre-emption in optical networks providing differentiated optical channel services.

\section{ACKNOWLEDGEMENT}

The author wish to thank Peter Braun his valuable support implementing some ILP models applied this study. This publication was supported by the Hungarian Italian Intergovernmental S\&T Cooperation Programme for 2004-2007, project reference number I-17/03.

\section{REFERENCES}

[1] S. Sengupta, R. Ramamurthy, "From Network Design to Dynamic Provisioning and Restoration in Optical Cross-Connect Mesh Networks: An Architectural and Algorithmic Overview", IEEE Network Magazine, July/August 2001

[2] L.Jereb, F.Unghváry, T.Jakab, "A Methodology for Reliability Analysis of MultiLayer Communication Networks", Optical Networks Magazine, 2 (2001), pp. 42-51.

[3] T. Jakab, H. Nakajima, H-M. Foisel, P. Szegedi, T. Zombori: Routing in translucent networks - Motivations and objectives of EURESCOM P1202 Project, NOC2002, Darmstadt, June 2002.

[4] H. Harai, M. Murata, H. Miyahara: Performance of Alternate Routing Methods in AllOptical Switching Networks, Proc. of the INFOCOM 97

[5] N. Andriolli, T. Jakab, L. Valcarenghi, P. Castoldi: Separate wavelength pools for multiple-class optical channel provisioning, Networks2004, Vienna 